\title{
Effectiveness of T'ai Chi and Qigong on Chronic Obstructive Pulmonary Disease: A Systematic Review and Meta-Analysis
}

\author{
Meng Ding, MD, ${ }^{1,2}$ Wei Zhang, MD, ${ }^{3}$ Kejian Li, MD, ${ }^{2}$ and Xianhai Chen, MD ${ }^{3}$
}

\begin{abstract}
Purpose: The purpose of this study is to determine the effects of Chinese traditional exercise such as t'ai chi and qigong (TCQ) on patients with chronic obstructive pulmonary disease (COPD).

Methods: All prospective, randomized, controlled clinical trials, published in English or Chinese and involving the use of TCQ by patients with COPD, were searched in 10 electronic databases from their respective inceptions to July 2012. The methodological quality of all studies was assessed using the Jadad score. The selection of studies, data extraction, and quality assessment were performed independently by two raters.

Results: In the results, 10 trials met the inclusion criteria and were reviewed. The meta-analysis demonstrated that compared with no exercise, TCQ had significant effects on 6-minute walk distance, forced expiratory volume in 1 second (FEV1), predicted FEV1 percentage, and St. George's Respiratory Questionnaire score. There were no significant differences in all outcomes between TCQ and other exercise training except 6-minute walk distance.

Conclusions: In conclusion, TCQ might be beneficial with respect to physical performance, lung function, remission of dyspnea, and quality of life in patients with COPD; however, caution is needed to draw a firm conclusion because of the low methodological quality of the included trials.
\end{abstract}

\section{Introduction}

C Hronic obstructive PUlmonary disease (COPD) is a common respiratory disease that impacts the health and quality of life of patients worldwide. According to a report of the World Health Organization, 64 million people had COPD throughout the world in 2004, and more than three million people died of COPD in $2005 .{ }^{1}$ An $8.2 \%$ overall prevalence of COPD in people $>40$ years of age was demonstrated by a cross-sectional survey in China between 2002 and 2004. ${ }^{2} \mathrm{~Pa}-$ tients with COPD frequently complain of dyspnea and exercise limitation and become trapped in a vicious cycle of inactivity, initiated by breathlessness. ${ }^{3,4}$ Exercise training, the important part of pulmonary rehabilitation, has been shown to improve dyspnea and health status and decrease health care use. $^{3}$

$T^{\prime}$ ai chi and qigong (TCQ) are both Chinese ancient forms of exercise with similar components such as movement of the extremities, meditation, and breathing control. TCQ has been practiced widely in recent decades to promote mental and physical health and to prevent chronic disease among individuals of all ages. ${ }^{5-7}$ Recently, TCQ was recommended for use in COPD rehabilitation, and some clinical trials were conducted to evaluate the effects of TCQ on patients with COPD. Therefore, it was necessary and timely to perform a systematic review and meta-analysis to summarize and critically evaluate clinical trial evidence for the effectiveness of TCQ as complementary therapy for COPD.

\section{Methods}

\section{Database source}

Databases searched from their respective inceptions to July 2012 included PubMed, EMBASE, Cumulative Index to Nursing and Literature Allied Health (CINAHL), Allied and Complementary Medicine Database (AMED), Physiotherapy Evidence Database (PEDro), REHABDATA, China National Knowledge Infrastructure (CNKI), Chinese Medical Database

\footnotetext{
${ }^{1}$ College of Physical Education, Shandong Normal University, Jinan, China.

${ }^{2}$ Shandong University of Traditional Chinese Medicine, Jinan, China.

${ }^{3}$ Affiliated Hospital of Shandong University of Traditional Chinese Medicine, Jinan, China.
} 
(CMD), Taiwan academic online, and the Cochrane Library. The following search terms were used: Tai Chi or Taiji or Tai Chi Chuan or Qigong or mind-body exercise and chronic obstructive pulmonary disease or COPD. Two raters performed the data searches (M.D. and K.L.).

\section{Inclusion criteria}

Trials were included if they (1) were randomized controlled trials; (2) included patients with COPD without restrictions of gender and age; (3) used Chinese traditional exercise such as $t^{\prime}$ ai chi or qigong or t'ai chi combined with qigong as the main intervention; (4) used nonexercise in control groups or other physical exercise training in comparison groups; and (5) published peer-reviewed articles or dissertations that used the English or Chinese language. Trials were excluded if they (1) used qigong that only involved breathing techniques and meditation but no physical activity; and (2) included patients with an acute exacerbation. Furthermore, observational studies, case series, and case reports were excluded.

\section{Data extraction and analysis}

All studies were reviewed and the data were extracted independently by two raters (M.D. and K.L.), and disagreements were resolved by seeking the opinion of the third rater (W.Z.). For each eligible study, the following information was extracted and recorded: (1) name of first author, (2) year of publication, (3) intervention and control group information, (4) duration of intervention, (5) sample size, (6) participants' demographic characteristics, (7) primary and all other, outcome measures, and (8) results. The mean change of outcome measures compared with a baseline was used to assess the differences between the intervention groups and the control or comparison groups. The pooled effect sizes and $95 \%$ confidence intervals (CIs) were calculated using the Cochrane Collaboration's software (Review Manager [RevMan] Version 5.1 for Windows, downloaded from http:// ims.cochrane.org/revman/download). Weighted mean differences (WMDs) and the random effects analysis model were used for continuous data. The $\chi^{2}$ test and the Higgins $\mathrm{I}^{2}$ test were used to assess heterogeneity.

\section{Quality assessment}

The methodological quality of all studies was assessed using the Jadad score. ${ }^{8}$ The Jadad score was calculated by assessing three criteria: description of randomization, blinding, and withdrawals; the score ranges from a minimum of 0 to a maximum of 5 points. Taking into account that patients cannot be blinded to the treatment, one point was given for blinding if the outcome assessor was blinded. The trial with a higher Jadad score had a higher methodological quality. Two raters performed the quality assessment independently (M.D. and K.L.). Disagreements were resolved by seeking the opinion of the third rater (W.Z.).

\section{Results}

\section{Search results}

A total of 507 potentially relevant articles were identified by the database searches. After 59 duplicates were removed, a total of 404 reviews and irrelevant articles were excluded through reading the titles and abstracts. Finally, by screening the full text, 10 trials (three published in English and seven published in Chinese) from 14 articles met the inclusion criteria and were reviewed (Fig. 1).

\section{Trials characteristics}

A total of 718 patients whose average age was more than 60 years participated in the 10 selected trials. The patients were recruited from outpatients or health care centers. Most of the trials used qigong as the experimental intervention; furthermore, two trials ${ }^{9,10}$ and one trial ${ }^{11}$ used $t^{\prime}$ ai chi and $t^{\prime} a i$ chi combined with qigong, respectively. All the included trials except one ${ }^{12}$ described the details involving the duration, frequencies, and session length of the interventions. Seven trials measured the outcomes for 3 months, ${ }^{9-15}$ while the other three trials measured outcomes for 6 months ${ }^{16,17}$ (Zhao, D.X. Rehabilitation of Modified Exercise Prescription for Elderly Patients with Stable COPD [in Chinese] 2011). The frequencies varied from one session per day and 2 days per week ${ }^{11,18}$ to two sessions per day and 7 days per week. ${ }^{15}$ One trial made the patients self-practice $t^{\prime}$ ai chi and qigong daily after the supervised sessions. ${ }^{11,18}$ Each session lasted from 20 to 60 minutes across the different trials. The interventions of control or comparison groups in all included trials were education, breathing technique, and walking combined with or without breathing technique. The patients of all trials received the usual pharmacological treatment in addition to the experimental intervention. The characteristics of the included trials are listed in Table 1.

\section{Methodological quality}

The range of Jadad score of the trials included in this review was from 1 to 4 (average score, 2.4). Seven trials were of low methodological quality;,12-16 (Zhao, D.X. Rehabilitation

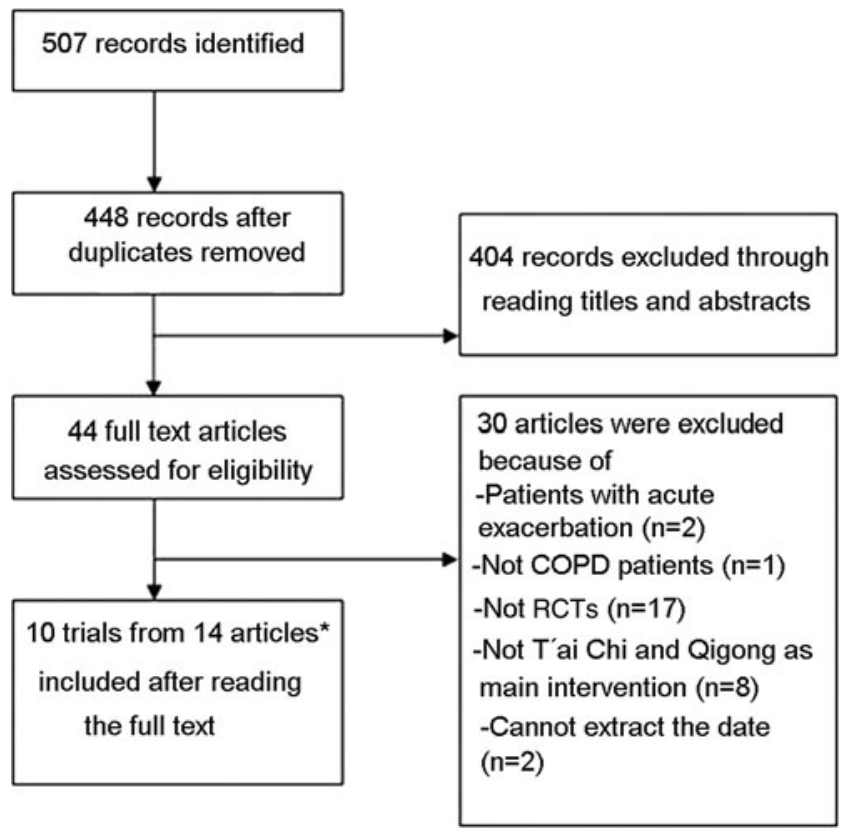

FIG. 1. Flowchart of articles selection process. ${ }^{*}$ There were seven articles that referred to the same trial (two, two, and three articles for each trial, respectively). COPD, chronic obstructive pulmonary disease; RCTs, randomized controlled trials. 


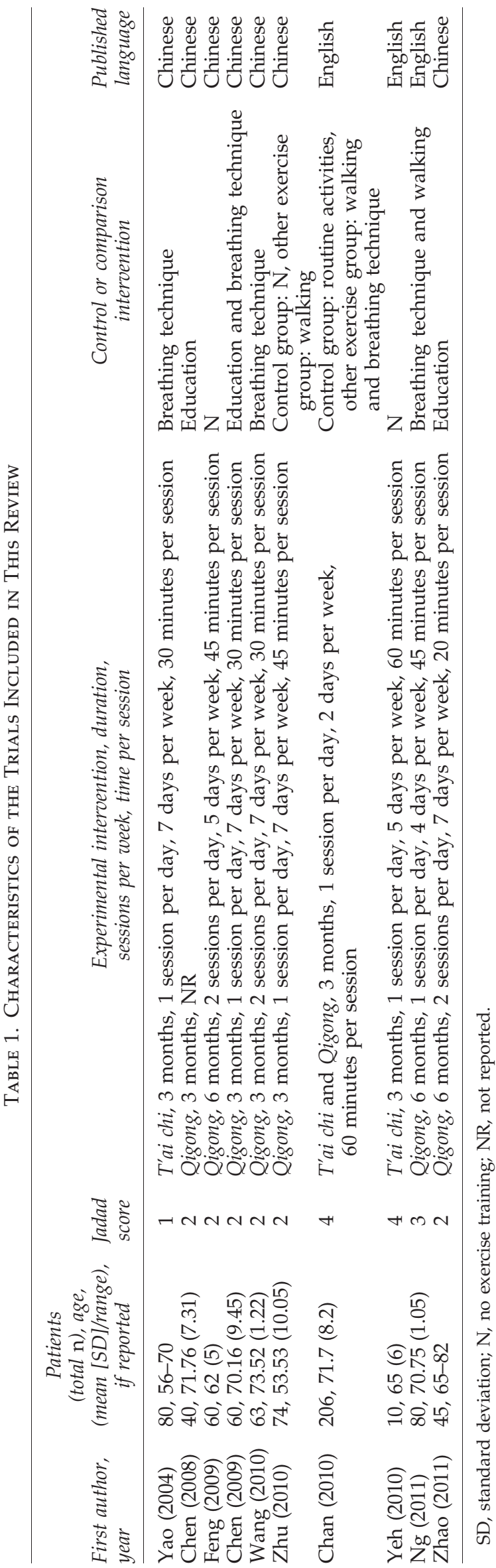

of Modified Exercise Prescription for Elderly Patients with Stable COPD [in Chinese] 2011) in contrast, the other three trials were of high methodological quality. ${ }^{10,11,17}$ All the trials described the methods of randomization, and only one $\mathrm{e}^{17}$ reported details on allocation concealment. Two trials ${ }^{10,11}$ mentioned the blinding to assessor for data collection. Three trials ${ }^{10,11,17}$ reported the using of intention-to-treat analysis.

\section{Outcomes}

Various outcomes including physical performance, lung function, dyspnea, and quality of life (QoL) were used in eligible trials of this review. The outcomes with same measurement in different trials were pooled in meta-analysis. The outcomes that could not be pooled in meta-analysis were used to calculate the effect size and are listed in Table 2.

\section{Physical performance}

\section{Six-minute walk test}

T'ai chi and qigong versus nonexercise. Of the 10 trials, seven $^{10,12,13,15,16,18}$ (Zhao, D.X. Rehabilitation of Modified Exercise Prescription for Elderly Patients with Stable COPD [in Chinese] 2011) ( $n=399$ patients) compared the distance of the 6-minute walk test (6MWT) between TCQ intervention and nonexercise. Two of seven trials ${ }^{10,12}$ could not be pooled in the meta-analysis because they did not provide the correct form of data. An attempt was made to contact the authors via e-mail to obtain the applicable data but failed. However, two trials reported that there was an improvement in 6minute walk distance (6MWD) after 3 months of $t^{\prime} a i$ chi or qigong exercise compared with the nonexercise group (Table $2)$. Five of seven trials showed heterogeneity when they were pooled in a meta-analysis $\left(\chi^{2}=26.89, \mathrm{I}^{2}=85 \%\right)$. The $6 \mathrm{MWD}$ effect size of one trial ${ }^{15}$ was obviously higher than the other four trials; furthermore, the other four trials showed homogeneity when this particular was removed. It was found that the trial showing the greatest improvement in 6MWD involved maintaining the exercise intensity of qigong within target range by monitoring the participants' heart rates; however, the other four trials did not report using any method to maintain the exercise intensity. Thus, a subgroup analysis was undertaken according to the particular exercise intensity in the intervention. Two subgroups-one named exercise intensity uncontrolled and another one named exercise intensity controlled-were established (Fig. 2). A pooled effect size of four trials ${ }^{13,16,18}$ (Zhao, D.X. Rehabilitation of Modified Exercise Prescription for Elderly Patients with Stable COPD [in Chinese] 2011) showed that TCQ intervention with uncontrolled exercise intensity could increase the length of 6MWD compared to nonexercise (WMD, 25.19 m; 95\% CI, 12.02 $38.37 \mathrm{~m} ; p<0.001)$, and qigong intervention with controlled exercise intensity showed a higher effect size in one trial ${ }^{15}$ (WMD, 110.40 m; 95\% CI, 80.52-140.28 m; $p<0.001$ ).

T'ai chi and qigong versus walking exercise. Three trials ( $n=272$ patients) estimated the effects of TCQ on 6MWD compared with walking exercise. A subgroup analysis was also undertaken because of the diversity of TCQ intervention (Fig. 3). A pooled effect size from the data of two trials ${ }^{17,18}$ demonstrated that TCQ intervention could improve the length of $6 \mathrm{MWD}$ compared to walking exercise when the 
Table 2. Outcomes Unable to Be Pooled in Meta-Analysis

\begin{tabular}{|c|c|c|c|c|c|}
\hline $\begin{array}{l}\text { First author, } \\
\text { year }\end{array}$ & Outcomes & $\begin{array}{l}\text { Experimental } \\
\text { intervention, } \\
\text { sample size }\end{array}$ & $\begin{array}{l}\text { Control or comparison } \\
\text { intervention, } \\
\text { sample size }\end{array}$ & Effect size, 95\% CI & $\begin{array}{l}\text { Favors } \\
\text { TCQ }\end{array}$ \\
\hline $\begin{array}{l}\text { Chen (2008) } \\
\text { Yeh (2010) }\end{array}$ & $\begin{array}{l}\text { Physical performance } \\
\text { 6-minute walk test } \\
\text { 6-minute walk test } \\
\text { Timed up and Go }\end{array}$ & $\begin{array}{l}\text { Qigong, } 21 \\
\text { T'ai chi, } 5\end{array}$ & $\begin{array}{l}\text { Education, } 19 \\
\mathrm{~N}, 5\end{array}$ & $\begin{array}{l}\text { NA } \\
\text { NA } \\
\text { NA }\end{array}$ & $\begin{array}{l}\text { Yes } \\
\text { Yes } \\
\text { Yes }\end{array}$ \\
\hline Yeh (2010) & $\begin{array}{l}\text { Dyspnea } \\
\text { UCSD SOB Questionaire } \\
\text { CRQ: Dyspneab }\end{array}$ & $T^{\prime} a i$ chi, 5 & $\mathrm{~N}, 5$ & $\begin{array}{l}\text { NA } \\
\text { NA }\end{array}$ & $\begin{array}{l}\text { Yes } \\
\text { Yes }\end{array}$ \\
\hline $\mathrm{Ng}(2011)$ & CRQ: Dyspnea ${ }^{b}$ & Qigong, 40 & $\begin{array}{l}\text { Walking and breathing } \\
\text { technique, } 40\end{array}$ & $0.04[-0.44,0.52], p=0.87$ & Yes \\
\hline Chan (2010) & $\begin{array}{l}\text { Quality of life } \\
\text { SGRQ: total score }\end{array}$ & $\mathrm{TCQ}, 70$ & $\begin{array}{l}\text { Walking and breathing } \\
\text { technique, } 69\end{array}$ & $-4.30[-9.54,0.94], p=0.11$ & Yes \\
\hline & $\begin{array}{l}\text { SGRQ: symptoms score } \\
\text { SGRQ: activity score } \mathrm{a}^{\mathrm{a}} \\
\text { SGRQ: impacts score }{ }^{\mathrm{a}}\end{array}$ & & & $\begin{array}{l}-2.40[-8.65,3.85], p=0.45 \\
-8.00[-14.74,-1.26], p=0.02 \\
-2.80[-8.73,3.13], p=0.35\end{array}$ & $\begin{array}{l}\text { Yes } \\
\text { Yes } \\
\text { Yes }\end{array}$ \\
\hline Yeh (2010) & $\begin{array}{l}\text { COPD Self-Efficacy score }{ }^{\mathrm{b}} \\
\text { CRQ: Fatigue } \\
\text { CRQ: Emotion } \\
\text { CRQ: Mastery } \\
\text { CR }\end{array}$ & $T^{\prime} a i$ chi, 5 & $\mathrm{~N}, 5$ & $\begin{array}{l}\text { NA } \\
\text { NA } \\
\text { NA } \\
\text { NA }\end{array}$ & $\begin{array}{l}\text { Yes } \\
\text { Yes } \\
\text { Yes } \\
\text { Yes }\end{array}$ \\
\hline $\mathrm{Ng}(2011)$ & SF-36 GH & Qigong, 40 & $\begin{array}{l}\text { Walking and breathing } \\
\text { technique, } 40\end{array}$ & $5.40[-2.68,13.48], p=0.19$ & Yes \\
\hline & $\begin{array}{l}\text { SF-36 } \mathrm{MH}^{\mathrm{b}} \\
\text { CRQ: Fatigue } \\
\text { CRQ: Emotion } \\
\text { CRQ: Mastery }\end{array}$ & & & $\begin{array}{c}-3.00[-10.81,4.81], p=0.45 \\
0.18[-0.30,0.66], p=0.46 \\
0.31[-0.15,0.77], p=0.19 \\
0.27[-0.21,0.75], p=0.27\end{array}$ & $\begin{array}{l}\text { No } \\
\text { Yes } \\
\text { Yes } \\
\text { Yes }\end{array}$ \\
\hline
\end{tabular}

${ }^{a}$ Lower score more favorable.

${ }^{\mathrm{b}}$ Higher score more favorable.

CI, confidence interval; TCQ, t'ai chi and qigong; UCSD SOB, University of California, San Diego, shortness of breath; SGRQ, St. George's Respiratory Questionnaire; COPD, chronic obstructive pulmonary disease; CRQ, Chronic Respiratory Questionnaire; SF-36, 36-Item Short Form Health Survey; GH, General Health; MH, Mental Health; NA, Not applicable; N, no exercise training.

exercise intensity was not controlled (WMD, $22.08 \mathrm{~m} ; 95 \% \mathrm{CI}$, 4.84-39.32 m; $p=0.01$ ). However, TCQ intervention with the controlled exercise intensity did not show any improvement of 6MWD compared with walking exercise in one trial ${ }^{15}$ (WMD, -16.70 m; 95\% CI, -43.58-10.18 m; $p=0.22$ ).

Timed up and go. One trial ${ }^{10}$ with a small sample size ( $n=10$ patients) evaluated the benefit on the timed up and go test. Regrettably, this trial provided the data in the form of median and range rather than mean and standard difference, so it was not possible to calculate the effect size of the data. According to the report of the trial, $t^{\prime} a i$ chi intervention could shorten the length of timed up and go compared with the nonexercise, but the difference between the two groups was not significant $(p=0.44)$.

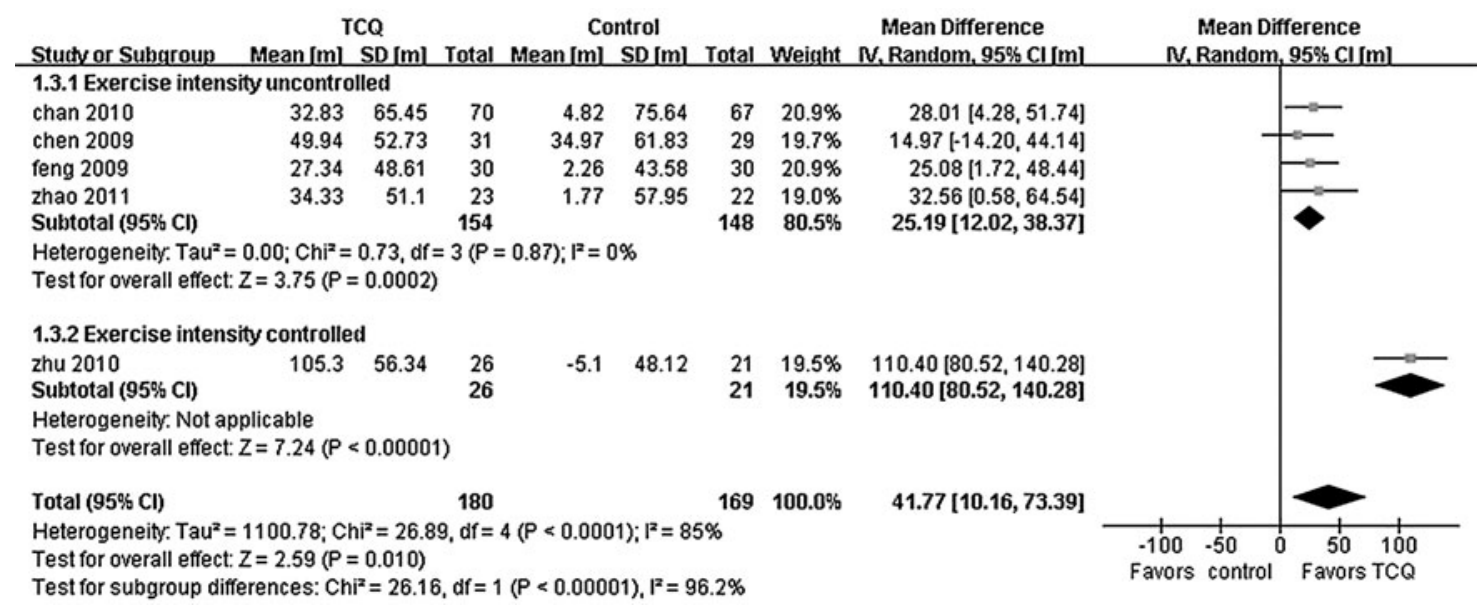

FIG. 2. Meta-analysis of 6-minute walking distance, t'ai chi and qigong (TCQ) versus nonexercise. CI, confidence interval; $\mathrm{SD}$, standard deviation. 


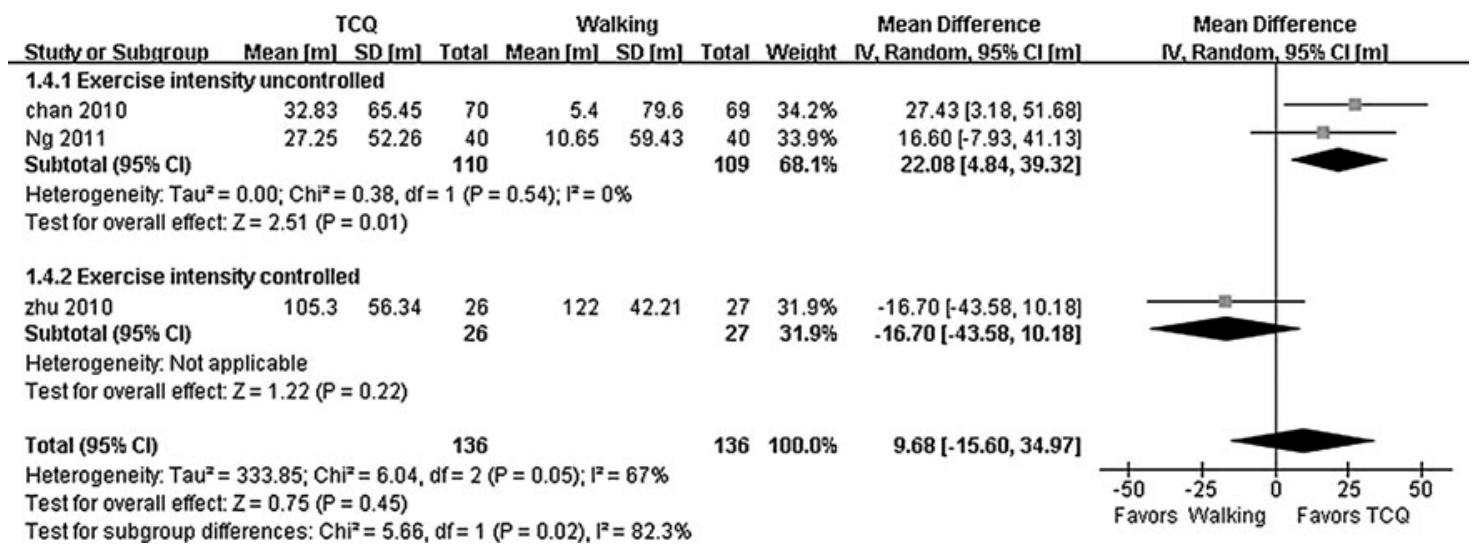

FIG. 3. Meta-analysis of 6-minute walking distance, t'ai chi and qigong (TCQ) versus walking. CI, confidence interval; SD, standard deviation.

\section{Lung function}

\section{Forced expiratory volume in 1 second}

T'ai chi and qigong versus nonexercise. Six trials ${ }^{13,15,16,18,19}$ (Zhao, D.X. Rehabilitation of Modified Exercise Prescription for Elderly Patients with Stable COPD [in Chinese] 2011) ( $n=389$ patients) estimated the effects of TCQ on the improvement in volume of FEV1 compared with nonexercise. We also undertook a subgroup analysis because of the heterogeneity that proceeded from the difference of details of intervention. A pooled effect size of five trials ${ }^{11,12,16,18}$ showed (Zhao, D.X. Rehabilitation of Modified Exercise Prescription for Elderly Patients with Stable COPD [in Chinese] 2011) that TCQ intervention without exercise intensity controlled could increase the volume of FEV1 compared to nonexercise intervention (WMD, 0.09 L; 95\% CI, 0.01-0.17 L; $p=0.02$ ), and qigong intervention with exercise intensity controlled had a higher effect size on improvement in volume of FEV1, but the difference was not significant (WMD, 0.27 liter; 95\% CI, $-0.05-0.59 \mathrm{~L} ; p=0.10)^{15}$ (Fig. 4).

Six trials ${ }^{9,13,15,16,19}$ (Zhao, D.X. Rehabilitation of Modified Exercise Prescription for Elderly Patients with Stable COPD [in Chinese] 2011) ( $n=332$ patients) estimated the effects of
TCQ on improvement of the FEV1 percentage predicted compared to nonexercise. A pooled effect size of five trials $^{9,13,16,19}$ (Zhao, D.X. Rehabilitation of Modified Exercise Prescription for Elderly Patients with Stable COPD [in Chinese] 2011) showed that TCQ intervention without exercise intensity controlled had a better effect on the FEV1 percentage predicted compared with nonexercise (WMD, 2.82\% predicted; $95 \% \mathrm{CI}, 0.29-5.34 \%$ predicted; $p=0.03$ ). In one trial, ${ }^{15}$ qigong intervention with exercise intensity controlled showed a higher effect size on improvement of FEV1 percentage predicted (WMD, 12.56\% predicted; 95\% CI, 5.25$19.87 \%$ predicted; $p<0.001$ ) (Fig. 5).

T'ai chi and qigong versus walking exercise. Two trials ${ }^{15,18}$ ( $n=192$ patients) compared the effects on the improvement of volume of FEV1 between TCQ and walking exercise. A trial with uncontrolled exercise intensity and the other one with exercise intensity controlled both showed that TCQ improved the volume of FEV1 compared with walking exercise; however, the differences were not significant (exercise intensity uncontrolled: WMD, 0.06 L; 95\% CI, $-0.07-0.19 \mathrm{~L}$; $\mathrm{p}=0.36$; exercise intensity controlled: WMD, $0.08 \mathrm{~L} ; 95 \% \mathrm{CI}$, $-0.21-0.37 \mathrm{~L} ; p=0.59$ ).

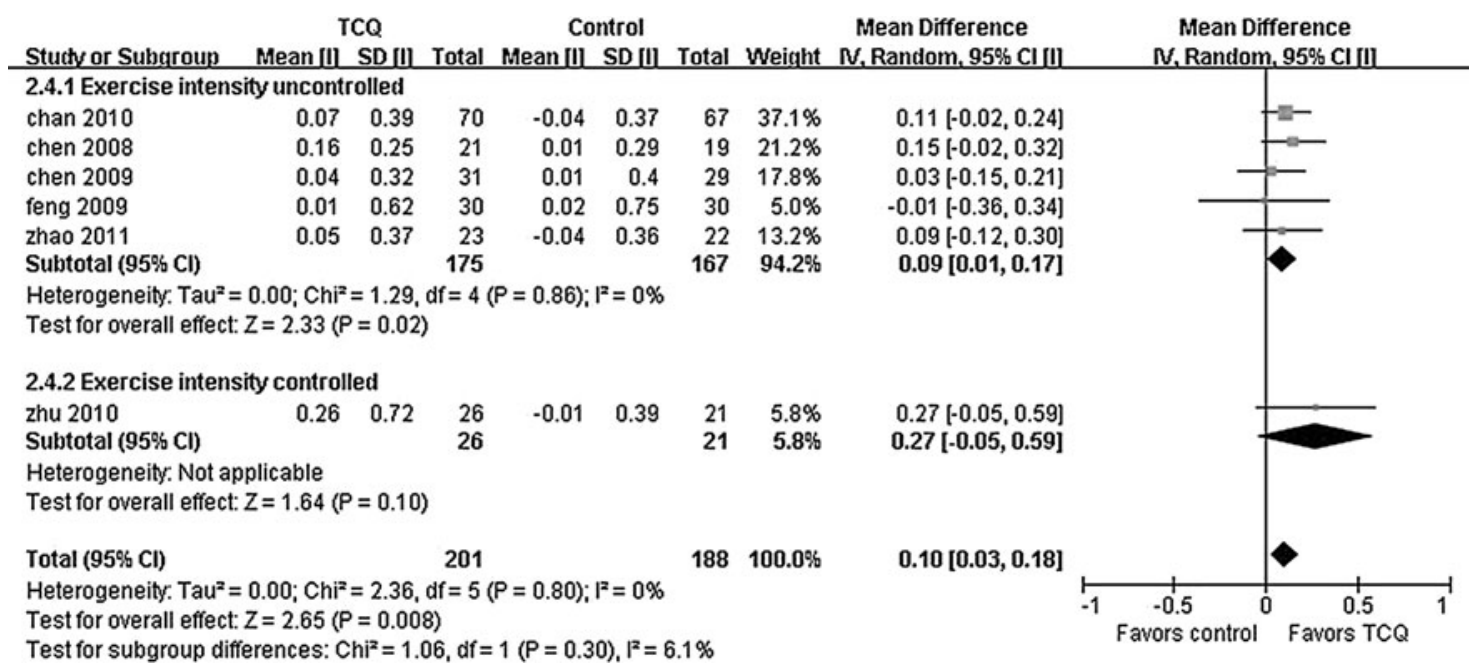

FIG. 4. Meta-analysis of the volume of forced expiratory volume in 1 second, t'ai chi and qigong (TCQ) versus nonexercise. $\mathrm{CI}$, confidence interval; SD, standard deviation. 


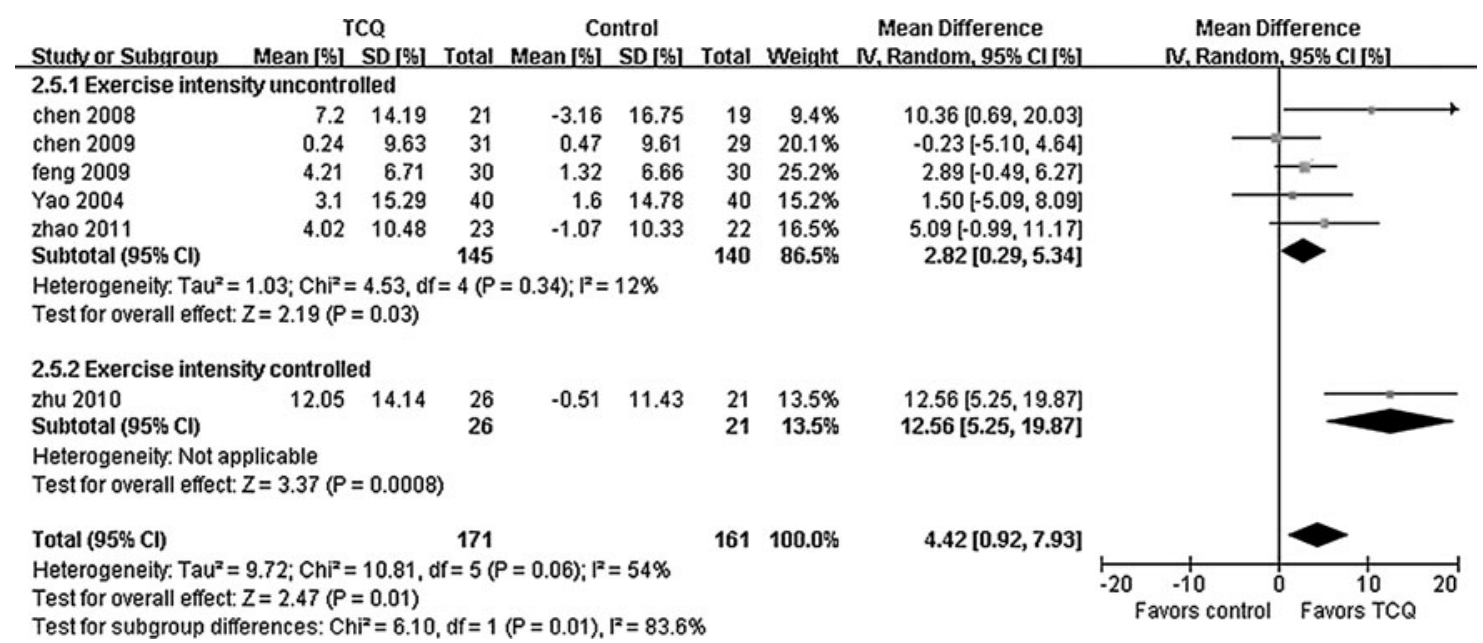

FIG. 5. Meta-analysis of forced expiratory volume in 1 second percent predicted, t'ai chi and qigong (TCQ) versus nonexercise. CI, confidence interval; SD, standard deviation.

\section{Dyspnea}

T'ai chi and qigong versus nonexercise. Two trials ${ }^{9,18}(n=217$ patients) evaluated the improvement of dyspnea using the Borg Scale; the pooled effect size showed that TCQ intervention had a positive effect on remission of dyspnea compared with nonexercise (WMD, $-0.82 ; 95 \% \mathrm{CI},-1.32$ to $-0.29 ; p=0.002)$. A trial ${ }^{10}$ ( $n=10$ patients) evaluated the improvement of dyspnea using the dyspnea section of the Chronic Respiratory Questionnaire (CRQ); it reported that the TCQ intervention showed an improvement in symptoms of dyspnea compared with nonexercise, but the difference was not significant. Moreover, an improvement in symptoms of dyspnea after the TCQ intervention was also reported in this trial using the Shortness of Breath Questionnaire (University of California, San Diego), but the difference was not significant with this questionnaire either (Table 2).

$T^{\prime}$ ai chi and qigong versus walking exercise. One trial ${ }^{18}$ $(n=139)$ reported that the TCQ intervention showed a greater improvement in symptoms of dyspnea compared with walking using the Borg Scale, but the difference was not significant (WMD, $-0.44 ; 95 \% \mathrm{CI},-0.91$ to $-0.03 ; p=0.07$ ). The improvement of dyspnea after the TCQ intervention was reported in another trial using the dyspnea section of CRQ, but the difference was not significant (Table 2).

\section{Quality of life}

$T^{\prime}$ ai chi and qigong versus nonexercise. Three trials ${ }^{11,14,20}$ ( $n=260$ patients) estimated the effects of TCQversusnonexercise using the St. George's Respiratory Questionnaire (SGRQ). The pooled effect sizeshowed that TCQhad a lowerSGRQscorethan nonexercise (SGRQ total: WMD, $-4.67 ; 95 \% \mathrm{CI},-8.21$ to -1.14 ; $p=0.009$; SGRQ symptoms: WMD, $-5.16 ; 95 \% \mathrm{CI},-10.15$ to $0.18 ; p=0.008$; SGRQ activity: WMD, -5.09 ; 95\% CI, $-10.46-$ $0.29 ; p=0.01$; SGRQ impacts: WMD, $-4.19 ; 95 \%$ CI, - 9.74-1.37; $p=0.05)$. The results of the COPD Self-Efficacy score and CRQ in one trial ${ }^{10}$ favored $t^{\prime}$ ai chi, but the data were not applicable and the differences were not significant (Table 2).

$T^{\prime}$ ai chi and qigong versus walking exercise. One trial ${ }^{11}$ ( $n=139$ patients) compared the differences in the effects on
SGRQ score of both TCQ and walking exercise. It was shown that TCQ could reduce the scores in each component of the SGRQ to a greater degree than walking exercise, but, apart from the activity score, the differences were not significant. Another trial ${ }^{17}$ ( $n=80$ patients) estimated the effects of qigong on CRQ compared with walking exercise. Differences in scores for each component of the questionnaire favored $q i$ gong, but the differences were not significant. Furthermore, the 36-Item Short Form Health Survey (SF-36) was used to evaluate the effect on QoL in this trial; the SF-36 score for general health favored qigong. In contrast, the SF-36 score for mental health favored walking exercise, but the differences were not significant (Table 2).

\section{Discussion}

In recent decades, 6MWT has been used as a simple, reliable, and valid assessment for exercise tolerance in COPD. $^{21-23}$ Some researchers have demonstrated that 6MWD is a better predictor of physical activity and mortality in patients with COPD than other methods of assessment. ${ }^{3,24,25}$ Many researchers have reported that TCQ might be beneficial for improving exercise tolerance in both healthy people and those with chronic diseases. ${ }^{26-30}$ According to the results of the present review, TCQ appears to demonstrate a greater improvement in physical activity such as exercise tolerance than intervention without exercise training. However, it cannot be confirmed that TCQ are the better complementary treatments for physical activity in COPD rehabilitation than other exercise training forms. A recent meta-analysis result also demonstrated that $t^{\prime}$ ai chi was not more beneficial for improving aerobic capacity in healthy or sick people than other physical exercises. ${ }^{31}$ Therefore, it is necessary to conduct trials with larger sample sizes and higher methodological quality to detect any advantages that TCQ might demonstrate for improvement in physical capacity compared with other exercise trainings in patients with COPD.

In this review, the pooled effect sizes showed that TCQ might be more beneficial for improving lung function in people with COPD than nonexercise. However, one researcher pointed out that the only successful intervention 
conclusively shown to attenuate the loss of lung function over time is smoking cessation. ${ }^{32}$ In addition, one metaanalysis of the effect of exercise training on lung function in patients with COPD demonstrated that the pooled effect of resistance training on FEV1 was neither clinically nor statistically significant. ${ }^{33}$ Furthermore, the follow-up durations of the trials in our review range from 3 to 6 months; the loss of lung function due to pathophysiological process of COPD might not be demonstrable in this short follow-up duration. Therefore, it remains unclear whether improvements gained in lung function due to TCQ can be maintained in the longer term. Further examination of the pulmonary functions in COPD patients over longer periods of time is recommended.

Dyspnea is the main symptom in COPD, and this results in inactivity and loss of QoL. ${ }^{34}$ Exercise training intervention may reduce the sensitivity to dyspnea. ${ }^{35}$ In the present review, a pooled effect from two trials shows that TCQ was more beneficial in reducing dyspnea in patients with COPD than nonexercise; however, there was no reduction in symptoms of dyspnea when TCQ was compared with nonexercise in another two trials. In another trial, it could not be confirmed whether or not TCQ was more effective at improving symptoms of dyspnea than other exercise training. Thus, firmer evidence needs to be provided through further clinical trials.

Some research demonstrated that TCQ might improve the QoL of the people with other chronic diseases such as heart failure, post-stroke, and cancer. ${ }^{36-38}$ In this review, TCQ may show a greater improvement in QoL in patients with COPD than nonexercise, based on the pooled result. However, it is unclear whether TCQ is more beneficial than other exercise trainings in improving QoL for COPD.

Some researches considered that the exercise intensity of TCQ is low to moderate through the estimation of the heart rate and energy expenditure of TCQ training. ${ }^{39-42}$ However, one review indicated that repeated high-intensity exercise stimulus might be beneficial for the patients with COPD due to the improvement of the cardiovascular function and the oxidative capacity of the skeletal muscle. ${ }^{35}$ Therefore, although TCQ might be suitable for the COPD patients who have low exercise capacity, it might not provide an optimal effect because of the insufficient exercise intensity. Therefore, we consider that if practitioners could try to enhance the exercise intensity of TCQ by using some means, the effects of TCQ on exercise capacity in patients with COPD might be more marked. In the present review, the researchers of one trial mentioned that they tried to keep the exercise intensity within target range by monitoring heart rate and the increase in movement of extremities; and improvement in 6MWD and FEV1 (percent predicted) in this trial were higher than other trials. The results of this trial might support our consideration; however, a firm conclusion could not be drawn because of the caution of bias due to the low methodological quality in this trial. Rigorous trials with larger samples should be conducted to determine the effectiveness of TCQ with controlled exercise intensity for COPD in the future.

There were several limitations to this systematic review. First, the low methodological quality of the included studies might affect the strength of the evidence. Especially, the poor-quality trials consistently reported positive results for all outcomes. Secondly, conference articles and unpublished trials were not searched, so publication bias is of concern. Thirdly, TCQ is popular in Japan and Korea, and some relevant studies may be published in Japanese or Korean literature. However, databases in Japan or Korea were not searched. Finally, most patients in the included trials are Chinese; they might like to participate in TCQ training because of the acceptance of traditional Chinese culture. It is possible that the willingness to participate in TCQ training is impacted by differences in national and ethnic cultures and customs.

\section{Conclusions}

TCQ might be beneficial with respect to physical performance, lung function, remission of dyspnea, and quality of life in patients with COPD; however, caution is needed about drawing a firm conclusion because of the low methodological quality of the included trials. More rigorous randomized controlled trials with larger sample sizes and longer treatment periods will be required in the future. Overall, TCQ are effective in COPD.

\section{Acknowledgments}

This review was supported by Taishan scholars project (ts20110819), independent innovation foundation of Shandong University (IIFSDU, 2012JC035) and health care Qigong project of Chinese General Administration of Sport. The authors thank Dr. Edwin Green for his help with translation.

\section{Authors' Contributions}

Meng Ding and Wei Zhang designed this review. Meng Ding and Kejian Li performed the article searches, data extractions, and meta-analysis. Meng Ding, Wei Zhang, and Xianhai Chen interpreted the results.

\section{Disclosure Statement}

No competing financial interests exist.

\section{References}

1. World Health Organization. Fact sheets: Chronic obstruct pulmonary diseases. Online document at: www.who.int/ mediacentre/factsheets/fs315/en/index.html. 2008. Accessed December 3, 2012.

2. Zhong N, Wang C, Yao W, et al. Prevalence of chronic obstructive pulmonary disease in China: A large, populationbased survey. Am J Respir Crit Care Med 2007;176:753-760.

3. Divo M, Pinto-Plata V. Role of exercise in testing and in therapy of COPD. Med Clin North Am 2012;96:753-766.

4. Singh S, Harrison S, Houchen L, Wagg K. Exercise assessment and training in pulmonary rehabilitation for patients with COPD. Eur J Phys Rehabil Med 2011;47:483-497.

5. Lee MS, Chen KW, Choi TY, Ernst E. Qigong for type 2 diabetes care: A systematic review. Complement Ther Med 2009;17:236-242.

6. Lee MS, Choi TY, Ernst E. Tai chi for breast cancer patients: A systematic review. Breast Cancer Res Treat 2010;120:309-316.

7. Wang WC, Zhang AL, Rasmussen B, et al. The effect of Tai Chi on psychosocial well-being: A systematic review of randomized controlled trials. J Acupunct Meridian Stud 2009;2:171-181. 
8. Jadad AR, Moore RA, Carroll D, Jenkinson C, et al. Assessing the quality of reports of randomized clinical trials: Is blinding necessary? Control Clin Trials 1996;17:1-12.

9. Yao Yp. Effect of tai chi chuan on chronic obstructive pulmonary disease [in Chinese]. J Chin Rehabil Theory Pract 2004;10:439-441.

10. Yeh GY, Roberts DH, Wayne PM, et al. Tai chi exercise for patients with chronic obstructive pulmonary disease: A pilot study. Respir Care 2010;55:1475-1482.

11. Chan AW, Lee A, Suen LK, Tam WW. Effectiveness of a Tai chi Qigong program in promoting health-related quality of life and perceived social support in chronic obstructive pulmonary disease clients. Qual Life Res 2010;19:653-664.

12. Zhang W, Chen J, Zheng G, et al. Influence of "Six-character" breathing method on exercise endurance of patients with chronic obstructive pulmonary disease in stable stage [in Chinese]. Chin Nurs Res 2009;23:2957-2958.

13. Chen J, Deng L. The effects of six-character formula respiratory gymnastics on quality of life in stable patients with COPD [J]. Chin J Natural Med 2009;24:944-945.

14. Wang Zw, Yang PL, Tang J, Huang Hy, et al. Effects of "Six Letters Formula" on quality of life and syndrome of traditional Chinese medicine inpatients with chronic obstructive pulmonary disease [in Chinese]. Shanghai J Tradit Chin Med 2010;55:54-57.

15. Zhu Y, Li N, Jin Hz. Effect on chronic obstructive pulmonary disease patient in stable condition by health qigong - wuqinxi exercise early treatment [in Chinese]. J Liaoning Univ Tradit Chin Med 2010;12:107-110.

16. Feng YC, Pan HS, Wen $X$, et al. The effects of Baduanjin exercise in old patients with stable COPD [in Chinese]. J New Chin Med 2009;41:36-37.

17. $\mathrm{Ng} \mathrm{BH}$, Tsang $\mathrm{HW}$, Jones $\mathrm{AY}$, et al. Functional and psychosocial effects of health qigong in patients with COPD: A randomized controlled trial. J Altern Complement Med 2011;17:243-251.

18. Chan AW, Lee A, Suen LK, Tam WW. Tai chi Qigong improves lung functions and activity tolerance in COPD clients: A single blind, randomized controlled trial. Complement Ther Med 2011;19:3-11.

19. ChenJ,Zhang W, Zheng G, etal. The application of six-character formula respiratory gymnastics in stable COPD rehabilitation [in Chinese]. J Fujian Univ Tradit Chin Med 2008;18:3-4.

20. Zhang W, Chen J, Zheng G. Influence of "Six-character" breathing method on exercise endurance of patients with chronic obstructive pulmonary disease in stable stage. Chin Nurs Res 2009;23:2957-2958.

21. Butland RJ, Pang J, Gross ER, et al. Two-', six-, and 12-minute walking tests in respiratory disease. Br Med J (Clin Res Ed) 1982;284:1607-1608.

22. Poulain M, Durand F, Palomba B, et al. 6-minute walk testing is more sensitive than maximal incremental cycle testing for detecting oxygen desaturation in patients with COPD. Chest 2003;123:1401-1407.

23. Chen $\mathrm{H}$, Liang BM, Tang YJ, et al. Relationship between 6minute walk test and pulmonary function test in stable chronic obstructive pulmonary disease with different severities. Chin Med J (Engl) 2012;125:3053-3058.

24. Spruit MA, Polkey MI, Celli B, et al. Predicting outcomes from 6-minute walk distance in chronic obstructive pulmonary disease. J Am Med Dir Assoc 2012;13:291-297.

25. Garcia-Rio F, Lores V, Mediano O, et al. Daily physical activity in patients with chronic obstructive pulmonary disease is mainly associated with dynamic hyperinflation. Am J Respir Crit Care Med 2009;180:506-512.

26. Li JX, Xu DQ, Hong Y. Changes in muscle strength, endurance, and reaction of the lower extremities with Tai Chi intervention. J Biomech 2009;42:967-971.

27. Xu DQ, Li JX, Hong Y. Effects of long term Tai Chi practice and jogging exercise on muscle strength and endurance in older people. Br J Sports Med 2006;40:50-54; discussion 50-54.

28. Tsai $\mathrm{YK}$, Chen $\mathrm{HH}$, Lin $\mathrm{IH}$, Yeh ML. Qigong improving physical status in middle-aged women. West J Nurs Res 2008;30:915-927.

29. Caminiti G, Volterrani M, Marazzi G, et al. Tai chi enhances the effects of endurance training in the rehabilitation of elderly patients with chronic heart failure. Rehabil Res Pract 2011;2011:761958.

30. Xu DQ, Hong Y, Li JX. Tai Chi exercise and muscle strength and endurance in older people. Med Sport Sci 2008;52:20-29.

31. Lee MS, Lee EN, Ernst E. Is tai chi beneficial for improving aerobic capacity? A systematic review. Br J Sports Med 2009;43:569-573.

32. Doherty DE. A review of the role of FEV1 in the COPD paradigm. COPD 2008;5:310-318.

33. Strasser B, Siebert U, Schobersberger W. Effects of resistance training on respiratory function in patients with chronic obstructive pulmonary disease: A systematic review and meta-analysis. Sleep Breath 2013;17:217-226.

34. Tsiligianni I, Kocks J, Tzanakis N, et al. Factors that influence disease-specific quality of life or health status in patients with COPD: A review and meta-analysis of Pearson correlations. Prim Care Respir J 2011;20:257-268.

35. Troosters T, Gosselink R, Janssens W, Decramer M. Exercise training and pulmonary rehabilitation: New insights and remaining challenges. Eur Respir Rev 2010;19:24-29.

36. Langhorst J, Häuser W, Bernardy K, Lucius H, et al. Complementary and alternative therapies for fibromyalgia syndrome. Systematic review, meta-analysis and guideline. Schmerz 2012;26:311-317. [in German]

37. Ding M. Tai Chi for stroke rehabilitation: A focused review. Am J Phys Med Rehabil 2012;91:1091-1096.

38. Mishra SI, Scherer RW, Geigle PM, et al. Exercise interventions on health-related quality of life for cancer survivors. Cochrane Database Syst Rev 2012;8:CD007566.

39. Lan C, Chen SY, Lai JS. The exercise intensity of Tai Chi Chuan. Med Sport Sci 2008;52:12-19.

40. Lan C, Chen SY, Wong MK, Lai JS. Tai Chi training for patients with coronary heart disease. Med Sport Sci 2008;52:182-194.

41. Hui SS, Woo J, Kwok T. Evaluation of energy expenditure and cardiovascular health effects from Tai Chi and walking exercise. Hong Kong Med J 2009;15(suppl 2):4-7.

42. Leung RW, McKeough ZJ, Peters MJ, Alison JA. Short-form Sun-style Tai Chi as an exercise training modality in people with COPD. Eur Respir J 2013;41:1051-1057.

Address correspondence to: Wei Zhang, MD Affiliated Hospital of Shandong University of Traditional Chinese Medicine 42 Wenhuaxi Road Jinan 250011

China

E-mail: 15666400659@163.com 